\title{
Integrable open boundary conditions for XXC models
}

\author{
D. Arnaudon* \\ Laboratoire d'Annecy-Le-Vieux de Physique Théorique LAPTH \\ B.P. 110, F-74941 Annecy-Le-Vieux cedex, France ${ }^{\ddagger}$ \\ and \\ Z. Maassarani ${ }^{\S}$ \\ Département de Physique, Pav. A-Vachon \\ Université Laval, Ste Foy, Qc, G1K 7P4 Canada ${ }^{9}$
}

\begin{abstract}
The XXC models are multistate generalizations of the well known spin- $\frac{1}{2} \mathrm{XXZ}$ model. These integrable models share a common underlying su(2) structure. We derive integrable open boundary conditions for the hierarchy of conserved quantities of the XXC models . Due to lack of crossing unitarity of the $R$-matrix, we develop specific methods to prove integrability. The symmetry of the spectrum is determined.
\end{abstract}

September 1998

LAPTH-695/98, LAVAL-PHY-22/98

Key words: Spin-chain, Integrability, Open Boundary Conditions

\footnotetext{
*Email address: arnaudon@lapp.in2p3.fr

†URA $1436 \mathrm{du}$ CNRS, associée à l'Université de Savoie

‡Work partially supported by European Community contract TMR FMRX-CT96.0012

$\S$ Email address: zmaassar@phy.ulaval.ca

『Work supported by NSERC (Canada) and FCAR (Québec).
} 


\section{Introduction}

Multistate generalizations of the spin- $\frac{1}{2}$ XXZ model were studied in [1]. These XXC models are not higher-harmonics generalizations like the spin- $j$ models. They have an underlying $s u(2)$ structure despite exhibiting features related to the $s u(n)$ Lie algebras. The transfer matrix for periodic boundary conditions was diagonalized by the algebraic Bethe Ansatz $[1,2]$. Comparing to the spin- $\frac{1}{2}$ XXZ model, the spectrum shows a large degeneracy as well as the existence of new eigenvalues in the case of closed boundary conditions.

In [3] the defining Hamiltonians of some multistate extensions of XXZ models were introduced. For open boundary conditions, their spectra were studied with direct methods, and without use of the $\mathcal{R}$-matrix approach. These models were then also studied in [4]. Here, to show integrability, we adapt and combine both approaches, along with a second new recursion proof. This is the crux of this work. This specific treatment is required due to an unusual feature of the XXC $\mathcal{R}$-matrix: its lack of crossing unitarity. This property is usually needed to show integrability for open boundary conditions. We start with a short introduction of the general $(\mathcal{K}, \mathcal{R})$ construction for integrable open boundary conditions. A modified construction is introduced which allows to prove the commutation of the transfer matrices at different spectral parameters. The action of the transfer matrix on the Hilbert space of the chain is made transparent using the recursion proof and the algebraic method. The latter method can be used to show the affine symmetry of the transfer matrix.

\section{Transfer matrices and local Hamiltonians: the stan- dard construction}

In this section, we recall the standard construction of local integrable spin chain Hamiltonian from a representation of the Yang-Baxter algebra and solutions of the reflection equations. We follow the approach and the equations of $[5,6,7,8]$.

The Yang-Baxter algebra reads

$$
\begin{aligned}
& \check{\mathcal{R}}_{i, i+1}(u) \check{\mathcal{R}}_{i+1, i+2}(u+v) \check{\mathcal{R}}_{i, i+1}(v)=\check{\mathcal{R}}_{i+1, i+2}(v) \check{\mathcal{R}}_{i, i+1}(u+v) \check{\mathcal{R}}_{i+1, i+2}(u), \\
& \check{\mathcal{R}}_{i, i+1}(u) \check{\mathcal{R}}_{j, j+1}(v)=\check{\mathcal{R}}_{j, j+1}(v) \check{\mathcal{R}}_{i, i+1}(u) \quad \text { for } \quad|i-j| \geq 2 .
\end{aligned}
$$

The $\check{\mathcal{R}}$-matrix with spectral parameter $u$ satisfies the inversion relation:

$$
\check{\mathcal{R}}(u) \check{\mathcal{R}}(-u)=\zeta(u)
$$

The matrix $\mathcal{R}$ is related to the matrix $\check{\mathcal{R}}$ by $\mathcal{R}=\mathcal{P} \check{\mathcal{R}}$, the operator $\mathcal{P}$ being the permutation map $\mathcal{P}: x \otimes y \mapsto y \otimes x$. Usually, $\mathcal{R}$ satisfies a crossing unitarity relation:

$$
\mathcal{R}_{12}(u+2 \rho)^{t_{1}} M_{1} \mathcal{R}_{21}(-u)^{t_{1}} M_{1}^{-1}=\zeta(u+\rho) .
$$

The reflection equations are given by

$$
\mathcal{R}_{12}(u-v) \mathcal{K}_{1}^{-}(u) \mathcal{R}_{21}(u+v) \mathcal{K}_{2}^{-}(v)=\mathcal{K}_{2}^{-}(v) \mathcal{R}_{12}(u+v) \mathcal{K}_{1}^{-}(u) \mathcal{R}_{21}(u-v)
$$


and

$$
\begin{aligned}
\mathcal{R}_{12}(-u+v) \mathcal{K}_{1}^{+}(u) M_{1}^{-1} \mathcal{R}_{21}(-u-v+2 \rho) M_{1} \mathcal{K}_{2}^{+}(v)= \\
M_{1} \mathcal{K}_{2}^{+}(v) \mathcal{R}_{12}(-u-v+2 \rho) M_{1}^{-1} \mathcal{K}_{1}^{+}(u) \mathcal{R}_{21}(-u+v) .
\end{aligned}
$$

If the monodromy matrix $\mathcal{T}(u)$ is given by

$$
\mathcal{T}(u)=\mathcal{R}_{0 L}(u) \mathcal{R}_{0 L-1}(u) \cdots \mathcal{R}_{01}(u),
$$

one defines the double row transfer matrix as

$$
\begin{aligned}
t(u)= & \operatorname{tr}_{0}\left(\mathcal{K}_{0}^{+}(u) \mathcal{T}(u) \mathcal{K}_{0}^{-}(u) \mathcal{T}(-u)^{-1}\right) \\
= & \zeta(u)^{-L} \operatorname{tr}_{0}\left(\mathcal{K}_{0}^{+}(u) \check{\mathcal{R}}_{L 0}(u) \check{\mathcal{R}}_{L-1, L}(u) \cdots \check{\mathcal{R}}_{23}(u) \check{\mathcal{R}}_{12}(u)\right. \\
& \left.\times \mathcal{K}_{1}^{-}(u) \check{\mathcal{R}}_{12}(u) \check{\mathcal{R}}_{23}(u) \cdots \check{\mathcal{R}}_{L-1, L}(u) \check{\mathcal{R}}_{L 0}(u)\right)
\end{aligned}
$$

Here $L$ is the number of sites.

With the relations given above (Yang-Baxter algebra, unitarity and crossing unitarity for $\check{\mathcal{R}}$; reflection equations for $\check{\mathcal{R}}$ and $\mathcal{K}$ ), one can prove that the transfer matrices for different values of the spectral parameters commute mutually, i.e.

$$
[t(u), t(v)]=0 \quad \forall u, v .
$$

The normalizations of $\check{\mathcal{R}}$ and $\mathcal{K}^{-}$are not fixed by their equations. We take for convenience $\check{\mathcal{R}}(0)=\mathcal{K}^{-}(0)=\mathbb{1}$. Defining an open chain Hamiltonian as

$$
\begin{aligned}
\left.H \equiv \frac{d t(u)}{d u}\right|_{u=0}-\left.\frac{d}{d u} \operatorname{tr}_{0} \mathcal{K}_{0}^{+}(u)\right|_{u=0}= & \operatorname{tr}_{0}\left(\mathcal{K}_{0}^{+}(0)\right)\left(2 \sum_{j=1}^{L-1} \mathcal{H}_{j, j+1}+\left.\frac{d}{d u} \mathcal{K}_{1}^{-}(u)\right|_{u=0}\right) \\
& +2 \operatorname{tr}_{0}\left(\mathcal{K}_{0}^{+}(0) \mathcal{H}_{L 0}\right)
\end{aligned}
$$

with

$$
\mathcal{H}_{j, j+1}=\left.\frac{d}{d u}\right|_{u=0} \check{\mathcal{R}}(u)_{j, j+1}
$$

one is ensured that it will commute with the set of transfer matrices indexed by the spectral parameter:

$$
[t(u), H]=0 \quad \forall u .
$$

In the precise case presented in the next section, the crossing unitarity (3) is missing for the proof of the commutation (9).

\section{The specific model}

For the XXC models $\check{\mathcal{R}}$ has the form $[1]$

$$
\check{\mathcal{R}}(u ; \gamma)=P^{(1)} \sin \gamma+P^{(2)} \sin (\gamma-u)+P^{(3)} \sin u
$$


where $\gamma$ is a 'quantum group' parameter and $u$ is the spectral parameter. Let $E^{a b}$ be the $n \times n$ matrix with a one at row $a$ and column $b$ and zeros otherwise. Let $n, n_{1}$ and $n_{2}$ be three positive integers such that $n_{1}+n_{2}=n$, and $A_{1}, A_{2}$ be two disjoint sets whose union is the set of basis states of $\mathbb{C}^{n}$, with $\operatorname{card}\left(A_{1}\right)=n_{1}$ and $\operatorname{card}\left(A_{2}\right)=n_{2}$. The operators $P^{(i)} \operatorname{read}$ :

$$
\begin{aligned}
P^{(1)} & =\sum_{a_{1} \in A_{1}} \sum_{a_{2} \in A_{2}}\left(E^{a_{2} a_{2}} \otimes E^{a_{1} a_{1}}+E^{a_{1} a_{1}} \otimes E^{a_{2} a_{2}}\right) \\
P^{(2)} & =\sum_{a_{1}, b_{1} \in A_{1}} E^{a_{1} a_{1}} \otimes E^{b_{1} b_{1}}+\sum_{a_{2}, b_{2} \in A_{2}} E^{a_{2} a_{2}} \otimes E^{b_{2} b_{2}} \\
P^{(3)} & =\sum_{a_{1} \in A_{1}} \sum_{a_{2} \in A_{2}}\left(x_{a_{1} a_{2}} E^{a_{2} a_{1}} \otimes E^{a_{1} a_{2}}+x_{a_{1} a_{2}}^{-1} E^{a_{1} a_{2}} \otimes E^{a_{2} a_{1}}\right)
\end{aligned}
$$

The model $\left(n_{1}=1, n_{2}=1\right)$ corresponds to the spin- $\frac{1}{2} \mathrm{XXZ}$ model whose integrable structure with open boundary conditions has already been studied [6].

The parameters $x_{a_{1} a_{2}}$ can be removed or, equivalently, set to one in the $\mathcal{R}$ matrix. Define a diagonal operator by

$$
F=\prod_{a_{1} \in A_{1}} \prod_{a_{2} \in A_{2}}\left(x_{a_{1} a_{2}}\right)^{\frac{1}{2}\left(E^{a_{2} a_{2}} \otimes E^{a_{1} a_{1}}-E^{a_{1} a_{1}} \otimes E^{a_{2} a_{2}}\right)}
$$

The $F$-twist on the $\mathcal{R}$-matrix $[9,10], \mathcal{R}^{\left(x_{a_{1} a_{2}}=1\right)}(u)=F \mathcal{R}^{\left(x_{a_{1} a_{2}}\right)}(u) F$, eliminates the twist parameters. For open boundary conditions, this results in a similarity transformation for the transfer matrix:

$$
\begin{aligned}
& t^{\left(x_{a_{1} a_{2}}=1\right)}(u)=X t^{\left(x_{a_{1} a_{2}}\right)}(u) X^{-1} \\
& X=\prod_{a_{1} \in A_{1}} \prod_{a_{2} \in A_{2}}\left(x_{a_{1} a_{2}}\right)^{\sum_{i<j} E_{i}^{a_{1} a_{1}} E_{j}^{a_{2} a_{2}}}
\end{aligned}
$$

where $E_{j}^{a_{i} a_{i}}$ is denotes the $E^{a_{i} a_{i}}$ matrix acting on site $j$, i.e. the occupation number of site $j$ by state $a_{i}$ (equal to 0 or 1 ). A similar transformation was defined in $[11,12]$.

Unless otherwise noted, the twist parameters $x_{a_{1} a_{2}}$ are then all taken equal to 1 and the superscript $\left(x_{a_{1} a_{2}}\right)$ is dropped. To simplify the notation we also take, without loss of generality, $A_{1}=\left\{1, \ldots, n_{1}\right\}$ and $A_{2}=\left\{n_{1}+1, \ldots, n_{1}+n_{2}\right\} . \check{\mathcal{R}}$ is taken as $(13)$ divided by $\sin (\gamma-u)$.

For $\left(n_{1}, n_{2}\right) \neq(1,1)$ the general procedure of section 2 fails almost from the beginning since the matrix $\check{\mathcal{R}}$ does not satisfy crossing unitarity. The partially transposed matrix $\mathcal{R}^{t_{1}}$ is not even invertible. This feature was also noticed in [13] where some Lax pairs were found. However, we can still define a set of commuting transfer matrices and an open chain Hamiltonian with local interaction, using information provided by the underlying spin- $\frac{1}{2} \mathrm{XXZ}$ model.

\section{Transfer matrices and local Hamiltonians: Modified construction}

Let $\tilde{\mathcal{K}}^{ \pm}$be diagonal solutions of the reflection equations for the XXZ model for which one has $M=1$ and $\rho=\gamma[6]$. 
We define $\mathcal{K}^{-}$by

$$
\begin{array}{lll}
\mathcal{K}_{i}^{-}=\tilde{\mathcal{K}}_{1}^{-} & \text {for } & 1 \leq i \leq n_{1}, \\
\mathcal{K}_{i}^{-}=\tilde{\mathcal{K}}_{2}^{-} & \text {for } & n_{1}+1 \leq i \leq n_{1}+n_{2} .
\end{array}
$$

This matrix $\mathcal{K}$, together with $\mathcal{R}$, is solution of the reflection equation (4). Explicitly,

$$
\begin{array}{ll}
\mathcal{K}_{i}^{-}=\frac{\cos (u+\xi)}{\cos (u-\xi)} \quad \text { for } \quad 1 \leq i \leq n_{1} \\
\mathcal{K}_{i}^{-}=1 \quad \text { for } \quad n_{1}+1 \leq i \leq n_{1}+n_{2}
\end{array}
$$

Here $\xi$ is an arbitrary complex parameter.

The corresponding boundary term then reads

$$
\left.\frac{1}{2} \frac{d}{d u} \mathcal{K}_{1}^{-}(u)\right|_{u=0}=-(\tan \xi) \cdot \sum_{a_{1} \in A_{1}} E_{1}^{a_{1} a_{1}}
$$

where the subscript of $E$ indicates that it acts on first site.

The definition of $\mathcal{K}^{+}$is slightly different:

$$
\begin{array}{rlrl}
\mathcal{K}_{i}^{+} & =\frac{1}{n_{1}} \tilde{\mathcal{K}}_{1}^{+} & \text {for } & 1 \leq i \leq n_{1} \\
\mathcal{K}_{i}^{+}=\frac{1}{n_{2}} \tilde{\mathcal{K}}_{2}^{+} & \text {for } & n_{1}+1 \leq i \leq n_{1}+n_{2} .
\end{array}
$$

Solutions for $\tilde{\mathcal{K}}^{+}$being given from those of $\tilde{\mathcal{K}}^{-}$by $\tilde{\mathcal{K}}^{+}(u)=M \tilde{\mathcal{K}}^{-}(-u+\rho)$, one has

$$
\begin{aligned}
& \mathcal{K}_{i}^{+}=\frac{1}{n_{1}} \cdot \frac{\cos \left(-u+\rho+\xi^{\prime}\right)}{\cos \left(-u+\rho-\xi^{\prime}\right)} \quad \text { for } \quad 1 \leq i \leq n_{1}, \\
& \mathcal{K}_{i}^{+}=\frac{1}{n_{2}} \quad \text { for } \quad n_{1}+1 \leq i \leq n_{1}+n_{2} .
\end{aligned}
$$

Here $\xi^{\prime}$ is another arbitrary complex parameter.

The corresponding boundary term will then be equal to

$$
\frac{\operatorname{tr}_{0}\left(\mathcal{K}_{0}^{+}(0) \mathcal{H}_{L 0}\right)}{\left.\operatorname{tr}_{0} \mathcal{K}_{0}^{+}(u)\right|_{u=0}}=\left(\tan \xi^{\prime}\right) \cdot \sum_{a_{1} \in A_{1}} E_{L}^{a_{1} a_{1}}
$$

where a term proportional to the identity has been dropped, and where the subscript of $E$ indicates that it acts on the last site.

The expressions we gave for the integrable boundary terms use formula (10). We will now prove that it provides an integrable spin chain Hamiltonian.

We still consider spectral dependent (double row) transfer matrices as defined in (8). Although our $\mathcal{R}$ matrix does not satisfy the crossing unitarity condition, we will prove that these transfer matrices commute for different values of the spectral parameters. We will give two proofs of this argument: one by recursion, the other using direct algebraic manipulations. 
We want to show that the transfer matrix is "order-preserving" within every set $A_{i}$. More precisely, $t(u)\left|a_{1}^{(1)}, a_{1}^{(2)}, a_{2}^{(1)}, a_{3}^{(1)}, a_{2}^{(2)}, \cdots\right\rangle, a_{j}^{(i)} \in A_{i}$, is a linear combination of states where $a_{j}^{(i)}$ is to the left of $a_{j+1}^{(i)}$ for all j's, and fixed $i=1,2$. The recursion proof for the action of the transfer matrix uses the diagonal form of the $\mathcal{K}^{ \pm}$matrices and the specific structure of the $\check{\mathcal{R}}$-matrix. To this end let momentarily $e_{k}$ be an operator $E^{c d}$ corresponding to the $k^{\text {th }}$ 'quantum' i.e. non-auxiliary space 0 , and define two types of operators for a chain of $i$ sites:

- $\left(e_{1} \cdots e_{i}\right)_{1}$ is order-preserving

- $\left(e_{1} \cdots e_{i}\right)_{2}^{a_{1} a_{2}}$ is such that $\left(e_{1} \cdots e_{i}\right)_{2}^{a_{1} a_{2}} e_{i+1}^{a_{2} a_{1}}$ is order-preserving for a chain of $i+1$ sites.

Below all non-auxiliary indices are suppressed. Let

$$
\Pi_{i}(u)=\mathcal{R}_{0 i}(u) \cdots \mathcal{R}_{01}(u) \mathcal{K}_{0}^{-}(u) \mathcal{R}_{01}(-u)^{-1} \cdots \mathcal{R}_{0 i}(-u)^{-1}
$$

We now show by induction that, for $i \geq 2, \Pi_{i}(u)$ has the following form:

$$
\begin{aligned}
\Pi_{i}(u) & =\sum_{c \in A_{1} \cup A_{2}} f_{i}(u) E_{0}^{c c}\left(e_{1} \cdots e_{i}\right)_{1} \\
& +\sum_{a_{1} \in A_{1}} \sum_{a_{2} \in A_{2}}\left(g_{i}(u) E_{0}^{a_{1} a_{2}}\left(e_{1} \cdots e_{i}\right)_{2}^{a_{2} a_{1}}+h_{i}(u) E_{0}^{a_{2} a_{1}}\left(e_{1} \cdots e_{i}\right)_{2}^{a_{1} a_{2}}\right)
\end{aligned}
$$

where all the $u$-dependence is carried by the functions $f_{i}, g_{i}$ and $h_{i}$. These functions may carry an index dependence but this is irrelevant to the proof. An explicit calculation using a diagonal but otherwise arbitrary $\mathcal{K}^{-}$matrix, and the explicit $\mathcal{R}$-matrix, shows that $\Pi_{2}(u)$ has the above form. Assuming (31) holds for a given $i$, one calculates $\Pi_{i+1}(u)$ using the explicit $\mathcal{R}$-matrix and find that $\Pi_{i+1}(u)$ also has the form (31), for $i+1$. This concludes the recursion. Finally, for $i=L$ and a diagonal $\mathcal{K}^{+}$, tracing $\mathcal{K}_{0}^{+}(u) \Pi_{L}(u)$ over the auxiliary space 0 shows that only terms of type 1 remain. We have thus proved that the transfer matrix is order-preserving. If in addition $\mathcal{K}_{a}^{ \pm}=\mathcal{K}_{b}^{ \pm}$, for all $(a, b) \in A_{i} \times A_{i}$, then the matrix elements are "color-blind". This means one can calculate the non-vanishing matrix elements, between a bra and a ket from the Hilbert space of the chain, by simply replacing in both states all states of type $A_{1}$ by one representative $\beta_{1}$ in this set, and similarly for $A_{2}$. This is clear since the functions $f_{i}(u), g_{i}(u)$ and $h_{i}(u)$ are now color-free.

We can now conclude that two transfer matrices $t(u)$ at different spectral parameters commute. Taking an arbitrary matrix element of $[t(u), t(v)]$, introducing the closure relation of the Hilbert space between the two matrices, using the above results reduces the matrix element to one for the XXZ system. The equal weighing of $\mathcal{K}^{ \pm}$within the two sets $A_{1}$ and $A_{2}$ ensures that the commutation relation is correctly reproduced. This method is cast below in an algebraic setting.

Let $N_{1}\left(\right.$ resp. $\left.N_{2}\right)$ be the number of states of set $A_{1}\left(\operatorname{resp} A_{2}\right)$ in a $L$-site state, i.e.

$$
\begin{aligned}
& N_{1}=\sum_{j=1}^{L} \mathbb{1}_{n_{1}+n_{2}}^{\otimes(j-1)} \otimes\left(\begin{array}{cc}
\mathbb{1}_{n_{1}} & 0 \\
0 & 0
\end{array}\right) \otimes \mathbb{1}_{n_{1}+n_{2}}^{\otimes(L-j)} \\
& N_{2}=\sum_{j=1}^{L} \mathbb{1}_{n_{1}+n_{2}}^{\otimes(j-1)} \otimes\left(\begin{array}{cc}
0 & 0 \\
0 & \mathbb{1}_{n_{2}}
\end{array}\right) \otimes \mathbb{1}_{n_{1}+n_{2}}^{\otimes(L-j)}
\end{aligned}
$$


We introduce two families of operators $J_{(r, s)}^{(i) a b}(i=1,2)$ acting on $L$-site states, defined as in [3] by:

- $J_{(r, s)}^{(i) a b}\left(N_{i}-r\right)=\left(N_{i}-r\right) J_{(r, s)}^{(i) a b}=0$, i. e. $J_{(r, s)}^{(i) a b}$ vanishes on states with a number of states of $A_{i}$ different from $r$.

- $J_{(r, s)}^{(i) a b}$ acts as $E^{a b}$ on the $s^{\text {th }}$ site (first site to the left) on which the state belongs to $A_{i}$. (Note that $s \leq r$ and that $a, b \in\left\{1, \ldots, n_{1}\right\}$ if $i=1$ and $a, b \in\left\{n_{1}+1, \ldots, n_{1}+n_{2}\right\}$ if $i=2$.)

One can write explicitly

$$
J_{(r, s)}^{(1) a b}=\sum_{\substack{f:\{1, \ldots, r\} \rightarrow\{1, \ldots, L\} \\ f \text { increasing }}} E_{f(s)}^{a b} \prod_{k=1}^{r} \mathbb{1}_{f(k)}^{(1)} \prod_{j \notin \operatorname{Im}(f)} \mathbb{1}_{j}^{(2)}
$$

with $\mathbb{1}^{(i)} \equiv \sum_{a \in A_{i}} E^{a a}$, and a similar definition for $J_{(r, s)}^{(2) a b}$.

We also define $\bar{J}_{(r, s)}^{(i) a b}$ as acting similarly on $L+1$ sites, including the auxiliary space 0 .

One can check the following properties:

$$
\begin{aligned}
& {\left[\bar{J}_{(r, s)}^{(i) a b}, \check{\mathcal{R}}_{j, j+1}\right]=0} \\
& {\left[\bar{J}_{(r, s)}^{(i) a b}, \check{\mathcal{R}}_{L 0}\right]=0} \\
& {\left[\bar{J}_{(r, s)}^{(i) a b}, \mathcal{K}_{1}^{-}\right]=0} \\
& {\left[\bar{J}_{(r, s)}^{(i) a b}, \mathcal{K}_{0}^{+}\right]=0}
\end{aligned}
$$

Hence,

$$
\left[\bar{J}_{(r, s)}^{(i) a b}, \mathcal{K}_{0}^{+}(u) \mathcal{T}(u) \mathcal{K}_{0}^{-}(u) \mathcal{T}(-u)^{-1}\right]=0
$$

Our aim is now to get rid of the auxiliary space 0 and to prove the commutation of $J_{(r, s)}^{(i) a b}$ (without bar) with $t(u)$. One can notice that, on site 0 :

- $\bar{J}_{(r, s)}^{(i) a b}$ acts only as $E^{a b}$ with $a, b$ both in $A_{1}$.

- $\mathcal{K}_{0}^{+}(u) \mathcal{T}(u) \mathcal{K}_{0}^{-}(u) \mathcal{T}(-u)^{-1}$ acts only as $E^{a a}$, or as $E^{a b}$ with $(a, b) \in A_{1} \times A_{2}$ or $(a, b) \in$ $A_{2} \times A_{1}$.

The matrix elements of the commutator (39), considered as an operator acting on site 0, can now be used to prove $\left[J_{(r, s)}^{(i) a b}, t(u)\right]=0$. Let us consider $i=1$ and take first $c \in A_{2}$.

$$
\begin{aligned}
0 & =\left.\left[\bar{J}_{(r, s)}^{(1) a b}, \mathcal{K}_{0}^{+}(u) \mathcal{T}(u) \mathcal{K}_{0}^{-}(u) \mathcal{T}(-u)^{-1}\right]\right|_{c c} \\
& =\left[\left.\bar{J}_{(r, s)}^{(1) a b}\right|_{c c},\left.\mathcal{K}_{0}^{+}(u) \mathcal{T}(u) \mathcal{K}_{0}^{-}(u) \mathcal{T}(-u)^{-1}\right|_{c c}\right] \\
& =\left[1 \otimes J_{(r, s)}^{(1) a b},\left.\mathcal{K}_{0}^{+}(u) \mathcal{T}(u) \mathcal{K}_{0}^{-}(u) \mathcal{T}(-u)^{-1}\right|_{c c}\right]
\end{aligned}
$$


Taking then $c \in A_{1}$, and $a \neq b \in A_{1}$

$$
\begin{aligned}
0 & =\left.\left[\bar{J}_{(r+1, s+1)}^{(1) a b}, \mathcal{K}_{0}^{+}(u) \mathcal{T}(u) \mathcal{K}_{0}^{-}(u) \mathcal{T}(-u)^{-1}\right]\right|_{c c} \\
& =\left[\left.\bar{J}_{(r+1, s+1)}^{(1) a b}\right|_{c c},\left.\mathcal{K}_{0}^{+}(u) \mathcal{T}(u) \mathcal{K}_{0}^{-}(u) \mathcal{T}(-u)^{-1}\right|_{c c}\right] \\
& =\left[\mathbb{1} \otimes J_{(r, s)}^{(1) a b},\left.\mathcal{K}_{0}^{+}(u) \mathcal{T}(u) \mathcal{K}_{0}^{-}(u) \mathcal{T}(-u)^{-1}\right|_{c c}\right]
\end{aligned}
$$

Summing both (42) and (45) over $c$, on gets

$$
\left[J_{(r, s)}^{(1) a b}, t(u)\right]=0
$$

The same holds for $J_{(r, s)}^{(2) a b}$. As in [3], one defines

$$
J_{r}^{(i)}\left(E^{a_{1} b_{1}}, E^{a_{2} b_{2}}, \ldots, E^{a_{r} b_{r}}\right)=\prod_{s=1}^{r} J_{(r, s)}^{(i) a_{s} b_{s}}
$$

with $r=1, \ldots, L$, and $J_{0}^{(i)}()$ such that $N_{i} J_{0}^{(i)}()=J_{0}^{(i)}() N_{i}=0$. One has

$$
\mathbb{1}=\sum_{r=0}^{L} \sum_{\left\{a_{1}, \ldots, a_{r}\right\} \in A_{i}^{r}} J_{r}^{(i)}\left(E^{a_{1} \beta_{i}}, E^{a_{2} \beta_{i}}, \ldots, E^{a_{r} \beta_{i}}\right) J_{r}^{(i)}\left(E^{\beta_{i} a_{1}}, E^{\beta_{i} a_{2}}, \ldots, E^{\beta_{i} a_{r}}\right)
$$

$\beta_{i}$ being a chosen element in $A_{i}$.

Let us then consider $t(u) t(v)$, multiplied on the left by $\mathbb{1}$ written as in (48) for each $i=1,2$. Using the commutation relations (46), one gets

$$
\begin{aligned}
t(u) t(v)= & \sum_{r_{1}=0}^{L} \sum_{\left\{a_{1}, \ldots, a_{r_{1}}\right\} \in A_{1}^{r_{1}}} \sum_{r_{2}=0}^{L} \sum_{\left\{b_{1}, \ldots, b_{r_{2}}\right\} \in A_{2}^{r_{2}}} \\
& J_{r_{1}}^{(1)}\left(E^{a_{1} \beta_{1}}, E^{a_{2} \beta_{1}}, \ldots, E^{a_{r_{1}} \beta_{1}}\right) J_{r_{2}}^{(2)}\left(E^{b_{1} \beta_{2}}, E^{b_{2} \beta_{2}}, \ldots, E^{b_{r_{2}} \beta_{2}}\right) \quad t(u) t(v) \\
& \times J_{r_{2}}^{(2)}\left(E^{\beta_{2} b_{1}}, E^{\beta_{2} b_{2}}, \ldots, E^{\beta_{2} b_{r_{2}}}\right) J_{r_{1}}^{(1)}\left(E^{\beta_{1} a_{1}}, E^{\beta_{1} a_{2}}, \ldots, E^{\beta_{1} a_{r_{1}}}\right)
\end{aligned}
$$

Under conditions (22-23) and (27-28), the factor $t(u) t(v)$ in the centre of the formula is then projected to an equivalent of the product $\tilde{t}(u) \tilde{t}(v)$ of the XXZ model with boundary term. Commuting back the operators $J$ and using again (48), one gets

$$
[t(u), t(v)]=0 .
$$

Although this result was obtained for $x_{a_{1} a_{2}}=1$, it holds for arbitrary parameters. This is obvious from the similarity relation (18).

To conclude this section we have found the action of the transfer matrix on states involving only one set $A_{i}$. We have done so directly and checked the order-preserving property for these 
states, which can serve as pseudo-vacua for a Bethe Ansatz diagonalization. For $c_{i} \in A_{1}$ one has:

$$
\begin{aligned}
t(u)\left|c_{1}, c_{2}, \cdots, c_{L}\right\rangle= & {\left[\tilde{\mathcal{K}}_{2}^{+}(u) \tilde{\mathcal{K}}_{1}^{-}(u) \frac{\sin ^{2} \gamma}{\sin ^{2}(\gamma-\lambda)} \cdot \frac{\left(\frac{\sin ^{2} \lambda}{\sin ^{2}(\gamma-\lambda)}\right)^{L}-1}{\frac{\sin ^{2} \lambda}{\sin ^{2}(\gamma-\lambda)}-1}\right.} \\
& \left.+\tilde{\mathcal{K}}_{1}^{+}(u) \tilde{\mathcal{K}}_{1}^{-}(u)+\tilde{\mathcal{K}}_{2}^{+}(u) \tilde{\mathcal{K}}_{2}^{-}(u)\left(\frac{\sin ^{2} \lambda}{\sin ^{2}(\gamma-\lambda)}\right)^{L}\right]\left|c_{1}, c_{2}, \cdots, c_{L}\right\rangle
\end{aligned}
$$

The equal weights choice made for the $\mathcal{K}_{i}^{ \pm}$was used but the parameters $x_{a_{1} a_{2}}$ are arbitrary. Thus all states $\left|c_{1}, c_{2}, \cdots, c_{L}\right\rangle, \forall c_{i} \in A_{1}$ share the same eigenvalue. For $c_{i} \in A_{2}$ the action of $t(u)$ is obtained from the above with the changes $\tilde{\mathcal{K}}_{1}^{ \pm} \longleftrightarrow \tilde{\mathcal{K}}_{2}^{ \pm}$. Again the same degeneracy hold. Note also that similar expressions exist for arbitrary $\mathcal{K}_{i}^{ \pm}$, with a sum over $A_{1}$ or $A_{2}$ in each of the three contributions.

\section{$5 \quad$ Symmetries}

A straightforward generalization of the methods of [3] shows that the $\left(n_{1}, n_{2}\right)$-XXC models have a large affine symmetry. One first shows that the operators $J_{(r, s)}^{(i) a b}$, which where seen to commute with the transfer matrix, generate the $L^{\text {th }}$ tensorial power of the quantum algebra $U_{q}\left(s l\left(n_{1}\right)\right) \otimes U_{q^{\prime}}\left(s l\left(n_{2}\right)\right)$, for arbitrary $q$ and $q^{\prime}$. This can then be interpreted as an affine symmetry given by: $U_{q}\left(\widehat{s l\left(n_{1}\right)}\right) \otimes U_{q^{\prime}}\left(\widehat{s l\left(n_{2}\right)}\right)$.

For arbitrary twist parameters $x_{a_{1} a_{2}}$ the above symmetry is unchanged. Its generators (and the $J$-operators) are however modified by the similarity relation (18).

Some generators of the affine symmetry take a particularly simple form when all parameters $x_{a_{1} a_{2}}$ are equal to each other, but not necessarily to one. The operators $E^{a_{i} b_{i}}=\sum_{j} E_{j}^{a_{i} b_{i}}$, $\left(a_{i}, b_{i}\right) \in A_{i} \times A_{i}, i=1,2$, then commute with the transfer matrix and generate a $g l\left(n_{1}\right) \otimes g l\left(n_{2}\right)$ sub-algebra of the affine algebra. This small symmetry is the one expected from simple considerations which apply also to the closed periodic XXC chains.

\section{Conclusion}

We have shown that it is possible to construct XXC Hamiltonians with integrable open boundary conditions, within the framework of reflection matrices. A modified construction was necessary due to the lack of crossing unitarity of the $\mathcal{R}$ matrix. All the models share the same eigenvalues as the $\left(n_{1}=1, n_{2}=1\right)$ or XXZ model, but the eigenvectors form representations of an affine quantum algebra. The methods used here should admit straightforward generalizations to the multi-states models of [2]. An analytical or algebraic Bethe Ansatz diagonalization can be carried out starting from the pseudo-vacua introduced above. Finally, despite the particular nature of the $\mathcal{R}$ matrix, it is tempting to look for a generalization of the crossing symmetry or crossing unitarity relation which would permit a proof of integrability 
along the lines of the standard construction. However such a generalization does not seem obvious.

\section{References}

[1] Z. Maassarani, "The XXC Models," Phys. Lett. A244 (1998) 160-164, solv-int/9712008.

[2] Z. Maassarani, "Multiplicity $A_{m}$ Models," preprint LAVAL-PHY-20/98, Eur. Physical J. B (in press), solv-int/9805009.

[3] F. Alcaraz, D. Arnaudon, V. Rittenberg, and M. Scheunert, "Finite Chains with Quantum Affine Symmetries," Int. J. Mod. Phys. A9 (1994) 3473-3496, hep-th/9307103.

[4] T. Hakobian and A. Sedrakian, "Spin chain Hamiltonians with affine $U_{q}(g)$ symmetry," Phys. Lett. B377 (1996) 250-254, hep-th/9506195.

[5] I.V. Cherednik, "Factorizing particles on a half line and root systems", Theor. Math. Phys. 61 (1984) 977-983.

[6] E. K. Sklyanin, "Boundary Conditions for Integrable Quantum Systems," J. Phys. A21 (1988) 2375-2389.

[7] L. Mezincescu and R.I. Nepomechie, "Integrability of Open Spin Chains with Quantum Algebra Symmetry," Int. Mod. Phys. A6 (1991) 5231, Addendum A7 (1992) 5657.

[8] J. R. Links and M. D. Gould, "Integrable systems on open chains with quantum supersymmetry," Int. J. Mod. Phys. B10 (1996) 3461-3480.

[9] N. Reshetikhin, Multiparameter quantum groups and twisted quasitriangular Hopf algebras, Lett. Math. Phys. 20 (1990) 331.

[10] A. Foerster, J. Links and I. Roditi, Integrable multiparametric quantum spin chains. J. Phys. A31 (1998) 687, cond-mat/9801175.

[11] S. Dahmen, Reaction-Diffusion processes described by three-state quantum chains and integrability, J. Phys. A: Math. Gen. 28 (1995) 905, cond-mat/9405031.

[12] D. Arnaudon, C. Chryssomalakos, and L. Frappat, "Classical and Quantum sl(1|2) Superalgebras, Casimir Operators and Quantum Chain Invariants," Journ. of Math. Phys. 36/10 (1995) 5262, q-alg/9503021.

[13] R. Yue and R. Sasaki, "Lax pair for $S U(n)$ Hubbard model," preprint YITP-98-5, cond-mat/9801193. 